\title{
XXVIII.
}

\section{Ueber einen Fall von progressiver Muskelatrophie.}

\author{
Von \\ Dr. Arnold Pick, \\ Assistenzarzt an der Irrenheilanstalt Wehnen (Oldenburg).
}

$\mathrm{D}$ er nachstehende Fall wurde von Herrn Professor Westphal beobachtet, welcher mir die Krankengeschichte zur Disposition stellte und das Rückenmark zur Untersuchung übergab.

Der ungewöhnliche Beginn des Falles, die Complication mit interstitieller Fettwucherung, welche geeignet war, das Bild der reinen progressiven Muskelatrophie zu trüben, der interessante mikroscopische Befund, der Gelegenheit bieten wird, eine Reihe von Aufstellungen kritisch zu beleuchten, dürften die Mittheilung desselben rechtfertigen.

Minna Schmuhl, 27 Jahre alt, Handarbeiterin, wurde am 1. September 1868 in die Charité aufgenommen, am 3. d. M. zur Nervenabtheilung verlegt. Die Mutter der Patientin starb vor 10 Jahren am Typhus, der Vater, der Potator gewesen, etwa 4 Wochen vor Aufnahme der Patientin an einer unbekannten Krankheit; von 10 Geschwistern der Patientin leben blos drei, diese sind gesund, überhaupt leugnet Patientin jede hereditäre Disposition. Bis zu ihrem 12. Lebensjahre will Patientin, mit Ausnahme leichter Erkrankungen, gesund gewesen sein, doch erzählt sie, dass sie erst zu 4 Jahren gehen gelernt. Vom 12. Jahre ab will sie epileptische Anfälle gehabt haben, die jedoch blos des Nachts, wenn Patientin schlief, ohne Aura auftraten, und vorubergingen, ohne dass sie erwachte; am folgenden Morgen hatte sie jedesmal die Zunge zerbissen und fühlte sich so matt, dass sie dann oft 2 Tage zu Bett bleiben musste; Kopfschmerz hatte sie dabei nicht; genauere Angaben über die Häufigkeit der Anfälle weiss Patientin nicht zu machen, doch glaubt sie alle 3 oder 4 Wochen einen gehabt za haben; während dieser Zeit will Patientin schwachsinnig geworden sein; im 14. oder 15. Jahre, zu welcher Zeit sich das jetzige Leiden in einer Gehstörung zuerst manifestirte, sollen die Anfalle ohne äussere Veranlassung sistirt haben, und damit auch die Intelligenz vollständig wiedergekehrt sein. Ueber ihr jetziges Leiden weiss Patientin fast 
gar nichts anzugeben, sie wurde auf dasselbe offenbar erst aufmerksam, als die Gehstörung schon ziemlich bedeutend geworden war; die Bewegungen der Arme sind seit etwa $4 \mathrm{~J}$ ahren gestört, ob und wann die Wadenmusculatur an Umfang zugenommen, ist nicht zu eruiren. Patientin behauptet sicher, nie menstruirt gewesen zu sein und auch nie molimina menstruationis gehabt zu haben. Aetiologisch ist nur zu ermitteln, dass Patientin immer kümmerlich gelebt, oft sogar Noth gelitten.

11. September 1868. Patientin ist mittelgross, gracil gebaut, dürftig ernäbrt, von bleicher Hautfarbe, die Schleimhäute ziemlich gut gefärbt; der Schädel im Verhältniss zur Körpergrösse etwas voluminös, jedoch symmetrisch und gut gebaut, Haarwuchs reichlich; kein Kopfschmerz; Anschlagen des Schädels nicht schmerzhaft. Das Gesichtsskelet ist leicht asymmetrisch, die linke Hälfte etwas grösser; die linke Augenbraue steht etwas höher, vielleicht in Folge einer Hautnarbe über derselben; die $\mathrm{Mm}$. frontales und corrugatores supercilii agiren gut; die Lidspalte beiderseits gleich weit, Augenschluss normal; die Iris beider Augen gleich, die Pupillen gleich, eng, Reaction derselben beiderseits prompt, die Bewegungen der Bulbi normal. Das Gehör der Patientin ist gut, die Ohrmuscheln normal. Die Zunge wird gerade herausgestreckt, zeigt keine Abnormität, Bewegungen derselben nach allen Richtungen gut ausführbar; die Gaumenbögen stehen in gleicher Höhe, heben sich beiderseits gleich gut; die Uvula sieht nach links; keine Sprachstörung; die Intelligenz der Patientin zeigt sich nurmal. Schon eine fluchtige Besichtigung lässt eine im Nacken beginnende, gegen den Thorax zunehmende Muskelatrophie erkennen, die namentlich die Muskeln des Schultergürtels betroffen zu hoben scheint; die rechte Schulter steht etwas böher als die der anderen Seite, die Ränder des Cucullaris markiren sich beiderseits ziemlich gleich; die Fossa supra- und infraspinata beiderseits tief eingesunken; die Schulterblätter sind flügelförmig vom Thorax abgehoben, die Muskeln längs der Wirbelsänle scheinen nicht atrophisch; weniger eingesunken sind die Fossa supra- und infraclavicularis; gut erhalten scheint die Musculatur der Oberextremitaten, doch fuhlt sich dieselbe etwas schlaff an; die Hant derselben besitzt wenig Panniculus, ist jedoch sonst normal, namentlich nicht marmorirt; beim Heben der Schultern kommt die rechte höher zu stehen, dabei treten offenbar mehr Muskeln in Action als beim Gesunden, indem wohl in Folge von Wirkung der Mm. rhomboidei eine starke Drehung des oberen, inneren Winkels der scapula nach der Medianlinie hin eintritt; die Annäherung des Schulterblattes an die Wirbelsäule gelingt gut. Der linke Arm kann nur bis zur Horizontalen gehoben werden, die Musculatur des rechten ist besser erhalten, und wird derselbe fast bis zur Verticalen erhoben; die übrigen Bewegungen der beiden Oberextremitäten werden gut ausgeführt, die grobe Kraft der Hände ist gleich, nicht auffallend schwach.

Patientin kann, auch ohne sich anzulehnen, sitzen, wagt jedoch dann, aus Furcht hinzufallen, keine seitliche oder Vorwärtsbewegung, obgleich dieselben ausfübrbar sind; hat sich Patientin nach vorn übergebeugt, so geschieht das Aufrichten durch Aufstützen der Arme; soll Patientin sich vom Stuble erheben, so bringt sie das nur unter Aufbieten aller Kräfte und Zuhilfenahme der Musculatur des Kopfes und der Arme zu Stande, ein Beweis für die hochgradige Schwäche der langen Strecker der Wirbelsäule und der Glutaei; hat 
sie einmal das Gleichgewicht gefunden, so kann sie frei stehen, bei leichter Unterstätzung auch auf einem Beine; sie geht aber nur äusserst unsicher, schleppend, mit hochgradiger, lordotischer Krümmung der Lendenwirbelsäule, mit nach hinten geneigtem Oberkörper, der bei jedem Schritte nach der Seite des Standbeins hinschwankt; die Musculatur des Beckens ist hochgradig beeinträchtigt, während die der Unterschenkel anscheinend besser conservirt ist; trotzdem kann Patientin gar nicht steigen, auch das Gehen ermüdet sie sehr bald; aufgefordert sich zu setzen, lässt sie sich einfach fallen, allein ist sie nicht im Stande in's Bett zu kommen; liegend kann sie den linken Schenkel im Hüftgelenke nicht heben, auch die Flexion im Kniegelenke wird nur dadurch ermöglicht, dass Patientin die Ferse auf der Unterlage an sich zieht; die Bewegung im Fuss- und in den Zehengelenken ist frei.

Rechts ist das Verhalten beiläufig das gleiche, doch agiren die Muskeln im Ganzen etwas kräftiger. Die Haut der Unterextremitäten zeigt keine $A b$ normität; während der Umfang der Oberschenkel dem Ernährungszustande der Patientin entspricht, scheint die Musculatur der Unterschenkel in mässigem Grade hypertrophisch, fühlt sich jedoch nicht besonders hart an, trotzdem eine leichte Contractur der Achillessehne deutlich zu fühlen ist. Im Bette kann Patientin sich nur schraubenartig umdrehen, mit Hülfe der Arme, was nach links besser gelingt, als nach der anderen Seite. - Die Sensibilität ist intact, über eigentliche Schmerzen klagt Patientin nicht.

Die Wirbelsänle zeigt ausser der beim Gehen sich bildenden Lordose keine Deformität; der Thorax, in seinen oberen Partien etwas schmal, erweitert sich gut; Brust- und Bauchorgane normal; Darmfunction normal. Introitus vaginae etwas eng, Hymen vorhanden, rund, lässt den Zeigefinger ohne Mühe passiren, portio vaginalis weich, orificium uteri ext. nicht zu fühlen.

Die Untersuchung mit mittelstarken faradischen Strömen ergiebt folgendes: Die obere Partie des rechten Cucullaris ziemlich gute Reaction, die untere desselben keine Reaction; der rechte Rhomboideus reagirt deutlich, ebenso der rechte Serratus ant., der rechte Sternocleidomastoideus, der rechte Triceps sowie die Muskeln des Vorderarmes und der Hand derselben Seite; herabgesetzt an derselben scheint die Reaction im Latissimus dorsi, Pectoralis major und Biceps; vom Plexus aus ist die Reaction eine gute. An der linken oberen Extremität scheinen die Verhältnisse schlechter zu sein, nur die obere Portion des Cucullaris und der Sternocleidomastoideus reagiren gut, leidlich der Serratus ant. und Deltoides, der Rhomboideus, Pectoralis, Latissimus dorsi, die untere Portion des Cucullaris nur minimal; die Reaction vom Plexus ans ist gut; ebenso am Vorderarm und an der Hand. - Die Bauchmusculatur reagirt gut, gar nicht die Glutaei, vielleicht in Folge des bedeutenden Panniculus; die Reaction an den Oberschenkeln, sowie an den etwas hypertrophischen Unterschenkeln schwächer; doch ist das Resultat der electrischen Untersuchung nicht zweifellos in Folge bedeutender Unruhe der Patientin; nur an den Unterschenkeln scheint die electrocutane Sensibilität herabgesetzt, indem Patientin bei Untersuchung derselben weniger klagt.

11. December 1868. Patientin klagt über Reissen im ganzen Körper, das namentlich die Extremitäten ruckweise durchziehe. 
April 1869. Trotz systematischer Galranisation des Sympathicus verschlechtert sich der Gang der Patientin; der Ernährungszustand ist gut.

August 1869. In der ganzen vorhergehenden Zeit Klagen aber eigenthümliche Schmerzen in den Beinen, Gefuhl von Taubsein, Sensibilität objectiv normal.

September 1869. Umfang der Vorderarme dicht unterhalb des Ellbogengelenkes rechts $201 / 2$, links $221 / 2 \mathrm{Ctm}$. Umfang der Waden an der grössten Circumferenz links 34 , rechts $32 \mathrm{Ctm}$. Umfang der Oberschenkel beiderseits $45 \mathrm{Ctm}$. Die Muskeln der Unterschenkel reagiren bei $11 \mathrm{Ctm}$. Abstand, die Peronaei bei $9 \mathrm{Ctm}$.

Der Gang der Patientin ist bedeutend verschlechtert, ohne Unterstützung kann sie nur wenige Schritte gehen, Treppensteigen ist unmöglich; in ihrem Gange bietet Patientin das Bild der Muskelpseudohypertrophie.

November 1869. Die allgemeine Ernährung der Patientin scheint kaum merklich verändert; beim Entkleiden friert sie in entschieden abnormer Weise, dagegen will sie nie, auch im Hochsommer nicht, geschwitzt haben, Patientin verbringt die meiste Zeit im Bette, wo sie, im Rücken unterstïtzt, sitzt; Stehen ermüdet sie bald, und muss sie sich dabei anlehnen; längere Strecken geht sie nur mit Mühe, immer in Furcht hinzufallen; dabei ist die Wirbelsäule lordotisch verkrümmt, die Beine schiebt Patientin mit geringer Beugung im Hüftgelenke und nur wenig erhobener Fusssohle vorwärts; sie vermag die Beine nicht so hoch zu heben, um eine Stufe zu ersteigen; beim Fallen sinkt Patientin in die Knie; liegt sie am Boden, so vermag sie sich nur mit fremder Hülfe zu erheben und muss sogleich in die oben beschriebene Stellung gebracht werden, in welcher allein sie stehen kann; im Bette unterstützt sitzend, kann sie nuı mit Hülfe eines am Fussende befestigten Strickes sich nach rorn beugen; die Beugung des Kopfes nach rückwärts geht nur mit Schwiprigkeit und unter Schmerz vor sich; besser, jedoch langsam, gelingen die übrigeu Bewegungen des Kopfes.

Die Halsmusculatur ist dünn, die Portionen des Sternocleidomastoideus füblt man durch die dünne Haut als zarte Stränge; bei Heben der Schultern scheint der rechte, wenngleich etwas dünnere, kräftiger zu wirken. Die Musculatur der Brust etwas dürftig, der Pectoralis mässig gut zu füblen, die Mammae sehr gut entwickelt; an den Bauchmuskeln nichts Abnormes; Pressen will Patientin gut können, bei der Untersuchung misslingt ein Versuch dazu. Der Umfang der linken Schulter ist grösser als der der rechten, ebenso ist der linke Ober- und Vorderarm voluminöser als der rechte; dabei haben die Arme eigenthümliche Contouren an der Ulnarseite, wodurch bei supinirten und gestreckten Armen ein stumpfer Winkel (mit der Oeffoung nach aussen) zu Stande kommt und zwar rechts stärker; hervorgerufen wird diese Erscheinung dadurch, dass die Differenz zwischen dem Umfange des oberen und unteren Endes des Oberarmes ganz bedeutend zu Gunsten des letzteren ausfällt; auch tritt dadurch die Atrophie der Schultern desto auffälliger hervor; die voluminösen Oberarme fühlen sich schlaff, fast fluctuirend an. Patientin vermag beide Arme kaum bis zur Horizontalen zu beben, den rechten etwas höher als den linken; dabei hebt sich der innere Rand der Scapula rom Thorax stark ab, der innere untere Winkel derselben geht nach innen oben, ăhnlich, wenn auch nicht in dem Masse wie bei der Serratuslähmung. Um mit der linken Hand das rechte Ohrläppchen zu ergreifen, beugt Patienten 
den Arm im Ellbogengelenke, und führt ihn in dieser Stellung, mit den Fingern am Nacken gleichsam fortkriechend, zum Ohre. Beugung und Streckung in Ellbogengelenke gehen beiderseits ohne Differenz, langsamer als normal, von Statten, können jedoch durch leichten Fingerdruck verhindert werden; die grobe Kraft der Arme beiderseits in hohem Grade, rechts vielleicht etwas mehr, herabgesetzt; Bewegungen und grobe Kraft der Hände und Finger gut; Sensibilität normal.

Die Haut der Unterextremitäten bietet nichts Auffälliges; der Umfang des linken Oberschenkels am oberen Patellarrande $36 \mathrm{Ctm}$., des linken Unterschenkels unterhalb der Patella 31/1/2, $14 \mathrm{Ctm}$. tiefer $31^{1} / 2,8 \mathrm{Ctm}$. oberhalb des Sprunggelenkes $23 \mathrm{Ctm}$; rechts beiläufig dieselben Masse. Nirgend an den unteren Extremitäten atrophische Stellen; die Waden reichlich entwickelt, fühlen sich elastisch an; mit Bestimmtheit lässt sich nicht sagen, ob sie un. verhältnissmässig stark sind, allein Patientin giebt an, dass ibr das Volumen derselben aufgefallen, und sie anfänglich sugar an eine Geschwulst gedacht. Beugung im Hüftgelenke gelingt nicht, bei dem Versuche dazu, rückt die Patella nach aufwärts, der Fuss stellt sich in Dorsalflexion; Ab- und Adduction gelingt nur bei aufliegender Ferse und geschieht mehr stossweise. Beugung im Knie geschieht nur höchst unvollkommen, etwa bis zu einem Winkel von 1200, wobei die Ferse immer aufliegt und abnorme Contractionen in der Wade hinzutreten; Streckung des passir gebeugten Kniees geschieht durch Fortschieben der Ferse, das durch abwechselnde Dorsal- und Plantarflexionen des Fusses unterstützt wird. Die grobe $\mathrm{Kraft}$ in den Unterextremitäten in hohem Grade herabgesetzt, am besten erhalten scheint sie in den Sprunggelenken; die Sensibilität ist sowohl für Berührung wie für Nadelstiche gut erhalten, etwas stärkerer Druck der Haut oder Musculatur wird schmerzhaft empfunden; Temperaturgefühl vollkommen intact.

Die faradische Erregbarkeit ist nur schwer zu prüfen, da schon schwache Ströme, an einzelnen Stellen selbst das einfache Aufsetzen der Electroden der Patientin angeblich Schmerz verursachen, und sie zu allerlei willkürlichen, störenden Bewegungen veranlassen. Die Flexoren der Hand sind gut erreg. bar, die Extensoren nur durch stärkere Ströme, links leichter bei indirecter Reizung; der Deltoides reagirt auf die gleichen Ströme nicht, Biceps bleibt zweifelhaft, Sternocleidomastoideus und Cucullaris (in seiner oberen Portion) reagiren bei mässigen Strömen; in den Wadenmuskeln ist keine Contraction zu erzielen, doch bleibt das Resultat zweifelhaft. Patientin giebt die ihren Zehen, Füssen und Unterschenkeln gegebenen Stellungen bei geschlossenen Augen richtig an.

Aus mehrfachen in der ersten Hälfte des Jahres 1870 gemachten Bemerkungen geht hervor, dass die Gebrauchsfühigkeit der Arme bedeutend abgenommen.

August 1870. Patientin kann sich nicht mehr allein ausziehen; auch das Gehen soll etwas schlechter sein. Die Differenz im Umfang der Arme ist noch immer vorhanden, die linke Schulter stark abgeflach'; den linken Arm hebt Patientin kaum bis zur Horizontalen. Umfang des linken Oberarmes $24^{1 / 2} \mathrm{Ctm}$., des rechten $22^{1} / 2 \mathrm{Ctm}$., Vorderarm links 21 , rechts $20 \mathrm{Ctm}$. Die Hände nach wie vor gut, keine Atrophie an ihnen bemerkbar. Der Hals dünn, $28 \mathrm{Ctm}$. im Umfange; Fettpolster an der Brust leidlich, die Musculatur 
derselben dem Gefühle nach duinn, namentlich scheint der Pectoralis bei $A b$ duction des Armes dünn. Die Schulterblätter sind auffallend beweglich; es ist der Patientin aufgefallen, dass ihr seit einem Jahre die Röcke herabfallen, es wäre, als hätte sie keine Hüften. Oberschenkel beiderseits gut entwickelt, rechts scheinen die Adductoren etwas besser; Beugung im Hüftgelenke unmöglich; Umfang der Waden an der dicksten Stelle beiderseits $33 \mathrm{Ctm}$.; Beugung und Streckung im Kniegelenke unmöglich; Bewegung der Fuss- und Zehengelenke frei. Sensibilität intact.

Ende März 1871. Patientin ist nicht mehr im Stande zu gehen, hebt unterstatzt die Beine sehr langsam; deutliche Atrophie betrifft jetzt namentlich die hinteren Nackenmuskeln, Supra- und Infraspinatus, Deltoides und Rhomboideus. Händedruck beiderseits schwach.

Mai 1871. Patientin erkrankt unter den Erscheinungen einer Pneumonie, hochgradig erschwerte Expectoration.

5. Mai. Exitus lethalis.

$$
\text { Section, 9. Mai. (Dr. Ponfick). }
$$

Ziemlich flacher, kurzer Breitschädel, von mittlerer Dicke und Schwere, mit persistenter Sutura frontalis. Dura mater ziemlich fest adhärent, masssig dick. Pia durchweg stark injicirt, von der Hirnsubstanz mässig leicht abziehbar; nach deren Abziehen erscheint die Hirnoberfläche blass rosig mit einer Anzahl feiner Blutpunkte; an der Oberfläche des rechten Hinterlappens circa 2 Zoll von der Längsspalte entfernt ein ganz verkalkter Cysticercus, ein eben solcher an dem den Hinterrand der hinteren Centralwindung begrenzenden Gyrus. Die graue Substanz, sowohl der Windungen, wie der Ganglien, durchweg sehr blutreich, hortensienfarbig; die weisse Substanz gleichfalls sehr blutreich, schlaff, weich, mässig feucht; die Ventrikel kaum erweitert.

Die Betrachtung des Körpers zeigt keine wesentliche Differenz zwischen beiden Körperhälften; die Formen sind an den Unterextremitäten ziemlich voll, die Oberextremitäten, namentlich an Oberarmen und an der Schultergegend verhältnissmässig mager; insbesondere ist die Gegend der Scapulae sehr abgeflacht, und lässt die Contouren der Knochen scharf hervortreten; am beträchtlichsten ist die Einsenkung an der Fossa infraspinata.

Das Unterhautfettgewebe am ganzen Körper sehr reichlich, intensiv gelb, sehr grosslappig, an vielen Stellen lässt sich kaum eine Grenze zwischen Fettund Muskelgewebe ziehen. Bei Betrachtung der Muskeln des Rumpfes zeigt ein Theil relativ normales Aussehen und zwar besonders die Nackenmuskeln, der Cucullaris, und die längs der Wirbelsäule gelegenen, längsverlaufenden Rackenmuskeln; doch ist der Longissimus dorsi in seinem unteren Theile gleichfalls stark degenerirt. Die Degeneration zeigt sich in einer theils röthlichgelben, theils graugelben, an den fortgeschrittensten Stellen in einer gelben Färbung, welche letztere vollständige Aehnlichkeit mit blassem Fettgewebe herstellt; ein Unterschied besteht nur darin, dass in den Muskeln die frahere Streifung deutlich zu unterscheiden ist. An der linken Oberextremität zeigt sich der Pectoralis in seinem claricularen Antheile mässig grauroth, im sternalen vollständig graugelb, sehr verdünnt, an manchen Stellen fast papierdünn; dagegen ist der ganze Deltoides gleichmässig verändert, vollständig graugelb; die Muskeln des linken Oberarmes zeigen eine mehr fleckige Ver- 
änderung, indem sich zwischen noch mehr rothen Inseln sehr ausgedehnte gelbe Stellen finden; je weiter nach unten desto zahlreicher werden die rothen Stellen. Sehr starke Veränderungen zeigen die drei Glutaei, sie sind in eine weissgelbe, vollständig dem Fettgewebe gleichende, sehr dünne Lage verwandelt; an den unteren Extremitäten zeigt sich die Veränderung am stärksten im Sartorius und Gracilis, während die Flexoren und der Quadriceps femoris noch ziemlich zahlreiche röthliche Reste zeigen. Die Waden sind ziemlich stark, der Gastrocnemius ist gesprenkelt, im oberen Theile vorwiegend gelb, im unteren roth, der Soleus vollständig in eine gleichmässig gelbe Masse verwandelt, welohe die alte, schiefe Streifung noch sehr deutlich zeigt. Die Bauchmuskeln gleichfalls in sehr vorgeschrittener Veränderung, besonders der Transversus; auch der Rectus abdominalis in seiner ganzen Länge schmutzig graugelb. Das Diaphragma, linkerseits mit der Milz, rechts mit der Leber verwachsen, beiderseits sebr dünn, seine Muskelsubstanz schlaff, ganz hellgrauroth. Der M. ileopsoas beiderseits in hohem Masse atrophisch, in seiner ganzen Ausdehnung intensiv gelb.

Am Räckenmarke und seinen Wurzeln von aussen keine Abnormität, die Substanz von mittlerer Consistenz und Blutgehalt, die graue Substanz ziemlich mager, im Brust- und Lendentheil der Centralcanal deutlich sichtbar, vielleicht etwas erweitert.

Herz ziemlich gross, die Wand des rechten Ventrikels etwas dicker, die Musculatur beiderseits ziemlich derb, links zeigt sich eine wesentliche Differenz zwischen der inneren und ausseren Schicht, indem letztere dunkler gefärbt und durch vielfache grüngelbe, theils weissliche Einlagerungen fleckig erscheint. Endocardium und Klappen normal.

Beide Lungen gross und schwer, besonders die Unterlappen; letztere beiderseits adhärent; die oberen sind lufthaltig, von geringem Blutgehalt, die unteren derb infiltrirt bis auf einen, links grösseren, rechts kleineren, ödematösen Abschnitt. Im Bereich der Infiltration erscheint das Gewebe schmutzig grauroth, die Schnittfäche etwas uneben durch zahlreiche, flach grubenartige Einsenkungen, nirgends körnig; in diese Grundfarbe eingesprengt eine Anzahl grüngelber, eiterähnlicher Stellen von unregelmăssig fleckigem Ansehen, welche in der Regel die centralen Abschnitte der Lobuli einnehmen; in dem dazwischen liegenden, schlaffen Gewebe sieht man ganz feine, gelbliche Streifen und Pünktchen. Aus den mittleren und kleinsten Bronchien entleert sich reichliche Menge röthlich gelblichen Fiters.

Milz zum Theil adhärent, Gewebe derb, dunkelroth; Follikel deutlich und gross. Nieren ziemlich derb, Rindensubstanz blutreich, Glomeruli durch feine rothe Pünktchen deutlich gezeichnet. Leber ebenfalls adhärent, Gewebe sehr blutreich, die Acini an der Peripherie gelblich graubraun, gross und deutlich.

Der Sexualapparat zeigt sehr ausgedehnte, feste Verwachsungen zwisehen allen seinen Theilen und zugleich zwischen diesen, der Blase und dem Mastdarm. Die Eierstöcke unverhältnissmässig gross, während der Uterus sehr klein, etwas nach rechts, in hohem Masse anteflectirt. Hymen noch vorhanden, die Scheide eng, mit reichlichem Schleimsecret, das Orific. uteri ext. sehr eng, punktförmig, Labia uteri klein, derb, Cervicalcanal leicht erweitert, Höhle des Uterus sehr klein, und kurz, enthält einige Tropfen einer schmutzigbraunen, dünnen Flüssigkeit, seine Schleimhaut stark geröthet, narbig. Uterus sonst 
sehr blutreich, im ubrigen sehr derb. Beide Ovarien mit sehr kurzem Ligamentum ovarii verseben, besonders rechts, Obertäche etwas höckerig, lässt rechts mehrere Corpora lutea und röthliche Cysten durchscheinen; anf dem Durchschnitt das Gewebe sehr blutreich, viele glattwandige Cysten enthaltend, an der Oberfäche des einen ein schiefriggelb gefärbtes Corpus lut., am medianen Umfange eine erbsengrosse, von graurother, derber Kapsellage umschlossene Höhle; mitten in dem anderen sehr blutreichen findet sich ein T-förmiges, schwefelgelbes Corp. lut.; die Oberfläche des linken Orarium böckerig durch rundliche, glattwandige Cysten, im Gewebe zahlreiche, derbe, harte, zum Theil verkalkte Corp. lutea.

Die mikroscopische Untersuchung der Muskeln wurde von Herrn Professor Cohnheim auf Wunsch des Herrn Professor Westphal ansgeführt. Die Notiz darüher lautet: „eine interstitielle Fettanhäufung mit gleichzeitiger einfacher Atrophie der Muskelfasern; von letzteren sind nur ganz wenig entartet, mithin derselbe Befund wie bei Griesinger's Muskelhypertrophie, indess auch genau derselbe, wie er sich in den fettigen Muskeln um ankylotische Gelenke, bei Amputationsstümpfen d. i. bei Inactivitätsatrophien findet. Ich habe mich mehrfach aberzeugt, dass bei allen diesen die Muskelatrophie meist einfach ist, dagegen zugleich eine exhebliche Fettanhäufung im interstitiellen Gewebe stattfindet.“

Die von Herrn Professor West phal gleich nach der Section vorgenommene mikroscopische Untersuchung der vorderen und hinteren Wurzeln ergab vollständige Intactheit derselben; mit diesem Befunde stimmte auch der an den intramedullaren Antheilen der Wurzeln. Die gleichfalls von Herrn Professor Westphal ausgeführte Untersuchung eines erbärteten Nerv. ischiadicus ergab nichts vom Normalen Abweichendes, namentlich keine Veränderung des Marks und keine auffallende Zahl schmaler Nervenröhren.

Die von mir gemachte

mikroscopische Untersuchung des gehärteten Rückenmarkes ergab:

Im Cervicaltheil zeigen sich die grossen Ganglienzellen der Vorderhörner allenthalben an Zahl vermindert, vielfach sind sie zu grösseren oder kleineren, unregelmässigen Klümpchen geschrumpft, die jedoch nicht immer stärker pigmentirt sind, als die noch nicht atrophischen Zellen; an diesen letzteren zeigt sich ein fast constantes Abgebrochensein der Fortsätze, zuweilen ist auch der Leib der Zelle entzwei gebrochen; ${ }^{*}$ ) der Kern jedoch ist selbst an bis zu seinem volumen herabgeschrumpften Zellen vollkommen intact, rund, durchsichtig, und nur in denjenigen Zellen, welche der Sitz hoch-

*) Ich will hier gleich Gelegenheit nehmen, den etwaigen Einwurf, dass das Alter des Präparates einen Theil der Veränderungen verschuldet, zu berichtigen, indem mich Untersuchungen bedeutend älterer Präparate, so einer 7 Jahre lang in doppeltchromsaurem Kali gelegenen grauen Degeneration der Hinterstränge, belehrten, dass erhärtete Rückenmarke, in schwächeren Lösungen aufbewahrt, auch nach Jahren keinen Schaden geliten haben; und wenn auch zuzugeben ist, dass die dadurch zuweilen bewirkte Sprödigkeit ein $A b$ brechen einzelner Fortsätze bewirkt, so muss doch das massenhafte Auftreten solcher Bilder anf den Krankheitsprocess bezogen werden, um so mehr, wenn dieselben mit den von anderen Autoren gefundenen übereinstimmen. 
gradiger Pigmentanhäufung sind, ist über den Zustand des Kernes nichts zu eruiren. Der Process der Atrophie der grossen Ganglienzellen ist in den beiden Vorderhörnern oft in verschiedenem Grade, besonders zu Ungunsten des linken Vorderhornes zu beobachten, doch zeigt sich keine durch ganze Schnittreihen fortlaufende Differenz. Am meisten betroffen ist der mediale Zellenstrang (nach $\mathrm{Henle}$ ), weniger der laterale, doch auch nicht selten beide in gleichem Masse.

Im Dorsaltheile findet sich hochgradiger Schwund der Ganglienzellen in beiden Vorderhörnern, besonders derjenigen des medialen Zellenstranges. Im Lendentheile unterscheiden sich die Ganglienzellen der Vorderhörner namentlich durch das vielfache Fehlen der Fortsätze und durch ein Dünnersein in der Fläche, weniger durch Schrumpfung; die Atrophie wird ersichtlich durch die bedeutend verringerte Zahl der Zellen. Während jedoch im Beginn der Lendenanschwellung in Bezug darauf keine erhebliche und constante Differenz zwischen beiden Vorderhörnern obwaltet, zeigt sich etwa einen Centimeter tiefer ein so auffälliges Ueberwiegen der Atrophie der Ganglienzellen des linken Vorderhornes, dass in demselben oft nur vereinzelte atrophische Ganglienzellen zu sehen sind, während die Zahl derselben im rechten Vorderhorne nicht wesentlich vermindert erscheint; allein auch die Zellen dieses Hornes zeigen nicht mehr das normale Aussehen; sie entbehren meist der Fortsätze, sind sehr dunn, wenig pigmentirt, was ihnen ein viel durchsichtigeres Wesen ertheilt, als dies normalen Zellen eigen ist, die selbst an den dünnsten Schnitten einen keineswegs schmächtigen Zellleib darbieten. Im mittleren Drittheile der Lendenanschwellung findet sich auch noch öfter die gleiche Differenz zwischen beiden Vorderhörnern, allein sie ist nicht mehr so constant; der Zustand der Ganglienzellen gleicht auch hier dem vorher beschriebenen; im untersten Lendenabschnitte tritt der Process der Ganglienzellenatrophie etwas zurück, es finden sich öfter Zellen, die als normal gelten könnten. Die weisse Substanz sowie die Neuroglia der grauen, sind durchgehends normal, bis auf eine eigenthümliche Bildung des Centralcanals, die hier selbstständig beschrieben werden soll.

Im Cervicaltheile war das Lumen des Canals nur selten vorhanden, vielmehr fanden sich an seiner Stelle die bekannten, von den Autoren vielfach beschriebenen Zellenanhäufungen, ohne dass jedoch die Ausdehnung derselben eine abnorm grosse wäre; das Lumen des Canals, wenn vorhanden, war mit Blutkörperchen, geronnenem Elutplasma, oder einem Gemische beider erfüllt. Im Dorsaltheile ist das Lumen des Canals überall vorhanden, zuweilen von Zellenanhäufungen erfüllt; die Zellenanhäufungen um denselben sind bedeutender als im Halstheile; am entschiedensten aber haben dieselben zugenommen im Lendentheile; der Centralcanal ist etwas erweitert zur Form eines Octogons, das sich sehr häufig wie oben ausgefüllt zeigt. An einem Präparate des oberen Theiles der Lendenanschwellung, etwa entsprechend jener Partie, in welcher sich eine so auffallende Differenz zwischen den Vorderhörnern zeigte, findet sich ein zweiter, deutlich mit Cylinderepithel ausgekleideter Centralcanal, der enger als der andere normal grosse und vor ihm liegende Canal ist; eine bedeutende Längenausdehnung konnte dieser accessorische Centralcanal nicht besitzen, da sich an der ganzen Schnittreihe aus dieser Gegend kein zweites Präparat desselben fand, und nur an einem derselben 
eine in der ihm entsprechenden Gegend gelegene dichtere Häufung der Zellen, als das ihm angehörige Ende gedeutet werden konnte. Die gleiche Bildung eines $z$ weiten Centralcanals fand sich an vielfachen Schnitten des unteren Lendentheils; der accessorische Canal war auch hier an Schnitten, die etwa der Mitte seiner Länge entsprachen, gleich dem anderen Canale; an anderen, die seinen Enden entsprachen, von ganz feinem Lumen, überall deutlich von Cylinderepithel ausgekleidet; die ganze Bildung unterschied sich von der vorher geschilderten wesentlich nur dadurch, dass hier die beiden Canäle neben einander lagen. An anderen Stellen des Lendentheils fand sich ein normaler Centralcanal vor.

Ueberblicken wir das in Folge mangelhafter anamnestischer An. gaben nur sehr unvollkommene Bild unseres Falles, so sehen wir als das einzige bekannte ätiologische Moment Noth und Armuth, über andere sonst so häufig vorkommenden Cansalmomente, namentlich über Heredität war nichts zu erfahren, und nur die eine aueh von Anderen hervorgehobene Thatsache, dass die progressive Muskelatrophie namentlich in solchen Familien auftritt, welche reich an Kindern sind, trifft hier za. Was den Beginn der Erkrankung betrifit, so könnte man sich Angesichts der Angabe, dass Patientin erst im 4. Jahre gehen gelernt, veranlasst sehen, diese Erscheinung, welche auch in anderen Fällen, besonders von Atrophia musculorum lipomatosa, von den Autoren berichtet und in Fällen, in welchen sich eine fortschreitende oder auch stationär bleibende Schwäche der unteren Extremitäten anschliesst, mit Recht als das erste Symptom der Erkrankung angesehen wird, auch in unserem Falle so zu deuten; allein da dieselbe nur einer uncontrollirten Angabe der Patientin entspringt, und eine zehnjährige Pause der Krankheit ohne irgend welche zurückbleibende, auch der Patientin auffällige Schwäche berechtigter Massen nicht angenommen werden kann, so wäre wohl der Beginn der Krankheit etwa in das 13. oder 14. Lebensjahr der Patientin zu verlegen, jedenfalls vor den Zeitpunkt, welchen Patientin angiebt, wenn man dem Umstande Rechnung trägt, dass der Process der Atrophie früher begonnen, als die überdies, wie sie selbst angiebt, damals schwachsinnige Patientin etwas von Störung ihrer Bewegungen merkte.

So interessant auch die Mittheilung über die im 12. Jahre beginnenden und mit dem Ausbruche der progressiven Muskelatrophie sistirenden epileptischen Anfälle ist, so erlaubt dieselbe doch keinen Einblick in den möglichen Zusammenhang mit dem Grundleiden, für welchen ich aus der Literatur nur den nenesten von 0 . Berger in der medicinischen Section der schlesischen Gesellschaft für vaterländische 
Cultur (Sitzung vom 23. Juli 1875) vorgestellten Fall anführen will, wo bei einem von Atrophia musculoram lipomatosa befallenen Knaben von 12 Jahren vom 3. bis 6. Jahre epileptische Anfälle auftraten, ohne lass irgend welche erbliche Disposition bestand. Nicht unmöglich wäre es übrigens, dass ein solcher Zusammenhang in unserem Falle wenigstens nicht besteht, die Anfälle vielmehr in Beziehung zu. bringen sind zu den bei der Section gefundenen zwei Cysticercen in der Hirnrinde. Ucber die mit den epileptischen Anfällen gleichen Schritt haltende psychische Schwäche darf man wohl nur Vermuthungen hegen, vielleicht handelt es sich um postepileptische Schwächezustände, welche Patientin als geistige Schwäche auslegte.

Durch den Beginn der Krankheit in den unteren Extremitäten, worüber bei den ganz präcisen Angaben der Patientin kein $Z_{w}$ eifel gestattet ist, clfaracterisirt sich der Fall als einer der selteneren, denn $\mathrm{Duchenne}$, dessen eigene Beobachtungen die der übrigen Autoren an Zabl weit überragen, will diese Art des Beginnes unter 159 Fällen nur zweimal gesehen haben; nach einer von Friedreich (Ueber progressive Muskelatrophie. Berlin 1873. S. 207) gemachten Zusammenstellung begann die Atrophie $27 \mathrm{Mal}$ unter 146 Fällen in den Beinen; unter dessen eigenen Fällen finden sich auch einzelne, und ausserdem führt er (i. c. S. 256) einen Fall von Erb an, der ebenso begonnen; Hammond (A treatise on diseases of the nervous system. pag. 666) sah diesen Beginn $8 \mathrm{Mal}$ in 29 Fällen, allein $\mathrm{Charcot}$ (Leçons sur les maladies du système nervenx. Paris 1874. III. part. pag. 202), der ihn für sehr selten bält, bezweifelt wohl mit Recht die klinisch berechtigte Diagnose einzelner der von $H$ ammond angeführten Fälle als reine progressive Muskelatrophien. - Eine Bestätigung der Angabe der Patientin, dass das Leiden in den Beinen begonnen, ist auch darin zu sehen, dass bei der Aufnahme derselben in das Krankenhans die Functionsstörung in den unteren Extremitäten am stärksten ausgesprochen war, und auch der nach anfwärts zum Schultergürtel gehende Weg der Atrophie klar vor Augen liegt. Leider fehlen für den nun folgenden Zeitraum fast alle Angaben, bis anf die, dass drei Jahre vor Aufnahme der Patientin die Schwäche in den Armen begonnen, und auch die spätere Mittheilung, das Patientin das Volumen ibrer Waden so auffällig fand, dass sie anfänglich an eine Geschwulst dachte, dürfte auf diese Zeit zu beziehen sein. Es wäre müssig, gestützt auf diese spärlichen Daten sich in Hypothesen über den genaueren Gang der Atrophie und der interstitiellen Fettwucherung einzulassen, allein der ascendirende Gang derselben, das spätere Hinzutreten der Pseudo. 
hypertrophie an den unteren Extremitäten, von der vielleicht noch anzunehmen ist, dass sie früher in stärkerem Masse ausgesprochen ge. wesen, als zur Zeit des Aufenthaltes der Patientin auf der Klinik, dürften als die gesicherten Facta aus diesem Zeitraume in Anspruch zu nehmen sein.

Dass die Annahme einer Volumensverminderung der früher durch iuterstitielles Fettgewebe geschwollenen Wadenmuskeln nicht ohne Begründung in der Erfahrung ist, beweist Friedreich's Fall III (1. c. S. 19), bei welchem Fried reic h selbst ein erheblich geringeres Volumen der neun Jahre früher von ihm in pseudohypertrophischen Zustande gesehenen Wadenmusculatur constatirte; eine gleiche Erfahrung berichten auch $\mathrm{Clarke}$ and Gowers (Med. chir. Transactions. Vol. 57. pag. 247).

Der weitere Verlauf der Krankheit vom Eintritte der Patientin in das Krankenhaus an, der progressive Gang der Krankheit, der sich theils in dem späteren Ergriffensein anfänglich noch intacter Muskeln, theils in dem vollständigen Verluste der vorher noch in mässigem Grade vorhandenen Function der atrophischen oder pseudohypertrophischen Muskelgrnppen ausdrückt, treten in der mitgetheilten Krankengeschichte so klar hervor, dass eine Commentirung derselben unnöthig, und ich mich auf eine Besprechung jener Punkte beschränken kann, welche entweder schon Bekanntes bestätigen, oder Neues bringen.

Bei Gelegenheit der Aufnahme der Patientin fiel eine leichte Asymmetrie der beiden Gesichtshälften auf, eine Erscheinung, welche auch in anderen Fällen schon die Aufmerksamkeit auf sich gelenkt hatte, (Hoffmann, Ueber sogenannte Muskelhypertrophie. Berlin. Dissertation 1867 und Roquette, Ueber sogenannte Muskelhypertrophie. Berlin. Dissertation 1868, bei Friedreieh, l. c S. 279 fg.); trotzdem halte ich es nicht für erlaubt, hieraus irgend welche Schlüsse zu ziehen, da dieselbe sich nicht auf den Schädel erstreckte, und eine genanere, darauf hinzielende Untersuchung eines grossen Krankenmateriales solche Deformitäten als nicht selten ergiebt. - Höchst characteristisch für die ungleichmässige Vertheilung der interstitiellen Fettgewebswucherung ist in unserem Falle die schon bei der ersten Untersuchung der Patientin so auffällige Differenz zwischen Function und Volumen der Muskeln, und wenn auch die Wadenmusculatur in besonderem Grade davon ergriffen war, so ist doch der vorliegende Fall geeignet, den Widerspruch zu bekräftigen, welchen Friedreich (l. c. S. 60) gegen die Ansicht Roberts (On wasting palsy. 1858. pag. 152) erhebt, dass bloss die Wadenmuskeln die interstitielle Fettwucherung erleiden; die 
Section unseres Falles ergab eine diffuse Verbreitung derselben und bestätigte die aus dem Symptome der hochgradig erschwerten Expec-

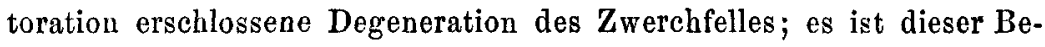
fund um so bemerkenswerther, als bisher nur wenige Fälle von derartiger Erkrankung des Zwerchfelles veröffentlicht sind; namentlich ist aber hervorzuheben, dass die Degeneration schon makroseopisch zu sehen war, während sie in Friedreich's Fällen (IV. und X.) erst durch die mikroscopische Untersuchung zu constatiren war; in dem dritten von ihm angeführten Falle (XXI) fehlt die anatomische Untersuchung, doch ist die Annahme der Degeneration des Zwerchfelles durch die Symptome der Endcomplication sehr wahrscheinlich. - Ebenso bemerkenswerth ist auch der Befund am Herzen und am M. sternocleidomastoideus, der bekanntlich nur sehr selten von der Krankheit crgriffen wird.

Hervorzuheben aus dem Befunde an den Muskeln ist nur noch die durch die ganze Krankengeschichte als rother Faden sich hindurchziehende, stellenweise nur angedeutete Differenz zu Ungunsten der linken Hälfte des Körpers, namentlich in Bezug auf die Functionsfähigkeit, welche in unserem Falle einen besseren Massstab abzugeben geeignet ist, als das scheinbare Volumen der Muskeln, und wenn sich auch im Rückenmarke kein völlig entsprechender Befund ergab, so glaube ich doch einen solchen im Lendentheile zu sehen (wobei ich zu bemerken nicht unterlassen will, dass das Rückenmark, um jede Voreingenommenheit zu vermeiden, noch vor genauerer Kenntnissnahme der Krankengeschichte untersucht wurde).

Von hohem Interesse ist die Angabe, dass das sogenannte Muske lg ef ühl vollkommen intact war, ein Beweis, dass dasselbe selbst bei hochgradiger Atrophie der Muskeln dennoch vollkommen functionirt, was der Ansicht, dass der Sitz desselben nicht in den Muskeln selbst oder deren Nerven zu suchen ist, eine neue Stiutze bietet. In Betreff der Sensibilität stimmt der vorliegende Fall mit den bisher darüber bekannten Thatsachen, indem dieselbe sich für Berührung, Schmerz und Temperatur vollkommen intact erwies; dafür finden sich in unserem Falle die von Charcot (l. c. pag. 201) für die progressive Muskelatrophie geleugneten spontanen Schmerzen, ebenso anch die von $\mathrm{Char-}$ cot für die sclérose laterale amyotrophique in Anspruch genommenen Schmerzen bei Druck anf die Muskeln (siehe Virchow-Hirsch, Jahresbericht für 1874. II. Bd. S. 169): beide Symptome führt jedoch auch Friedreich (1. c. S. 247) als nicht gar seltene an. Auffallender Weise fehlen im vorliegenden Falle die fibrillären Contractionen der 
Muskeln, ein sonst so häufig vorkommendes Symptom der progressiven Muskelatrophie, und könnte dafür wohl die von Friedreich (1. \&. S. 242) gegebene Erklärung Statt haben, dass schon frühzeitig die Complication mit Pseudohypertrophie eintrat, und dadurch sowie durch die sich hänfig damit verbindende diffuse Lipomatose, ein Punkt anf welchen zuerst W. Müller (Beiträge zur pathologischen Anatomie und Physiologie des Rückenmarkes, 1871.) die Aufmerksamkeit lenkte, die vielleicht dennoch vorhandenen fibrillären Zuckungen der in die Tiefe gedrängten Muskelfibrillen nicht zor Beobachtung kommen.

Noch treten uns bei unserer Patientin eine Reihe von Symptomen entgegen, welche gewöhnlich als vasomotorische bezeichnet werden; so finden wir die bei der progressiven Muskelatrophie so häufige Erscheinung verzeichnet, dass Patientin sehr leicht friert, dagegen ist ausdrücklich als fehlend angegeben die sonst nicht ungewöhnliche Marmorirung der Haut; ganz im Widerspruche jedoch mit den bisher gemachten Angaben über excessive Schweissbildung (Frommann, Fall aus Leubuscher's Klinik, Deutsche Klinik 1857. No. 33, 34 und Friedreich's Fälle IV und V.) steht die hier verzeichnete Thatsache des vollständigen Fehlens derselben, selbst im Hochsommer, was jedenfalls zur Vorsicht in der Deutung der bisherigen Erfahrungen mahnen muss.

Die Section bestätigte durch den mikroscopischen Befnnd die während des Lebens angenommene interstitielle Fettgewebswucherung; auch der Rückenmarksbefund ergab die in allen neveren Fällen vorgefundenen Veränderungen an den grossen Ganglienzellen der Vorderhörner, deren Beschreibung nur in wenigen unwesentlichen Punkten von den bekannten Schilderungen der Autoren abweicht; so fand sich der Kern der atrophischen Ganglienzellen immer, soweit er überhaupt noch gesehen werden konnte, vollkommen intact, während von Ley den, Charcot u. A. ein gleichzeitiges Schrumpfen desselben beschrieben wird; doch hatte ich Gelegenheit mich in einem von Herrn Professor Westphal untersuchten Falle von Tabes mit Veränderungen an den Gelenken, welcher neben der grauen Degeneration der Hinter- und Seitenstränge Atrophie der grossen Ganglienzellen der Vorderhörner darbot, von der Intactheit des Kernes derselben zu überzeugen. - Die verschiedenartigen Befunde an den Ganglienzellen, welche unser Fall in verschiedenen Höhen des Rückenmarkes darbot, zeigen, dass wir vorläufig noch nicht in der Lage sind, die verschiedenen Stadien des Processes zu schematisiren, und man vergebliche Mühe hätte, den vorliegenden Befund etwa in das von Clarke aufgestellte Schema hinein- 
zuzwängen. Die in Betreff des Centralcanals gemachten Beobachtungen sind kaum geeignet, Clarke's Ansicht, (Philos. Transactions 1859. P. I. pag. 455) der eine gleiche Bildung beobachtete, wie sie sich hier am Lendentheile vorfand, und dieselbe für Canalbildung innerhalb des Centralcanals hält, zu stützen, vielmehr glaube ich, dass es sich um einen schon embryonal angelegten, doppelten Centralcanal in den an Stelle des Centralcanals vorhandenen Zellenanhäufungen handelt.

Fragen wir nun an der Hand des Befundes, um welche Krankheit es sich hier handelt, so kann es kaum zweifelhaft sein, dass wir einen Fall von progressiver Muskelatrophie vor uns haben, der sich schon frühzeitig mit einer interstitiellen Fettgewebswucherung combinirte, und dadurch geeignet war, das Bild einer Atrophia musculorum lipomatosa vorzuspiegeln; ergab anch die von Cohnheim gemachte Untersuchung der Muskeln den für die letztere schon von Griesinger Billroth (Archiv für Heilkunde. VI. Bd. 1865. S. 1) durch Excision eines Muskelstückchens am Lebenden festgestellten Befund, so kann hierin doch kein Grund gegen die progressive Muskelatrophie gesehen werden, indem bekanntlich die interstitielle Fettwucherung keineswegs eine für die Psendohypertrophie characteristische Veränderung ist, sondern auch bei Kinderlähmung, namentlich aber schon in frühen Stadien der progressiven Muskelatrophie auftreten kann, wie dies Friedreich in seinem Falle V. (l. c. S. 28) durch Harpunirung am Gastrocnemins nachgewiesen. Es muss nach den neueren Aufstellungen $\mathrm{Ch}$ arcot's (Leçons sur les maladies du système nerveux. III.) zugegeben wcrden, dass man zwei Formen der Atrophia-musculorum lipomatosa zu unterscheiden habe; eine, welche als interstitielle Fettgewebswucherung blos eine Complication der gewöhnlichen progressiven Muskelatrophie bildet und bei der Rückenmarksuntersuchung Atrophie der grossen Ganglienzellen der Vorderhörner, den für die progressive Muskelatrophie massgebenden Befund, ergiebt, und eine reine Form der Lipomatosis musculorum luxurians, welche einen negativen Befund am Rückenmarke ergiebt. (Charcot, Note sur l'état anatomique des muscles et de la moëlle épinière dans un cas de Paralysie pseudohypertrophique. Arch. de phys. norm. et path. 1872. pag. 229). Der Fall von Cohnheim (Verhandlungen der Berliner medic. Gesellschaft 1866. S. 191), in welchem es sich um die complicatorische Form handelte, ergab zwar negativen Rückenmarksbefund, allein schon von W. Müller (l. c. Fall II.) warden Zweifel angeregt, ob das Rückenmark dabei in gehärtetem Zustande untersucht worden, was unbedingt in allen den Fällen nothwendig ist, in denen über die Natur der Ganglien* 
zellen der Vorderhörner etwas ausgesagt werden soll. - Charcot's Ansicht entgegen sieht Friedreich (l. c. S. 310), ausgehend von der Ansicht der myopathischen Natur der progressiven Muskelatrophie, consequenter Weise in der Pseudohypertrophie blos „eine durch gesteigerte Intensität der Krankheitsanlage und durch gewisse Besonderheiten des Kindesalters modificirte Form der progressiven Muskelatrophie." Noch anders steht W. Müller (l. c. Fall II.) der Frage gegenüber, indem er die reine Form der Atrophia musculorum lipomatosa lengnet und nur die combinirte Form zulässt, jedoch im Gegensatze zu Friedreich die Muskelatrophie von der Atrophie der Ganglienzellen in den Vorderhörnern ableitet; allein dieser Standpunkt wird durch den seither veröffentlichten Fall von $\mathrm{Charcot}$ unbaltbar; noch existirt ein positiver Rückenmarksbefund in einem Falle von Barth (Archiv für Heilkunde XII. 1871. S. 121), allein derselbe rangirt unter die von Charcot aufgestellte sclérose latérale amyotrophique, bei der die Muskelatrophie eine secundäre ist (1. c. S. 262. S. auch VirchowHirs ch, Jahresbericht für das Jahr 1874. II. Bd. S. 167); einen neuesten von Clarke und Gowers veröffentlichten Fall (Med. chir. Transactions. Vol. 57. p. 247) kann ich anch nur als mit Pseudohypertrophie combinirte Muskelatrophie betrachten.

Haben wir nun bisher die Atrophic der Ganglienzellen als unterscheidendes Merkmal für die progressive Muskelatrophie angenommen und damit auch indirect die primäre Natur derselben, so liegt es uns nun ob, die Gründe, welche Friedreich in den Capiteln IV. und V. seines grossen Werkes dagegen anführt, als dessen Tendenz er ja ausdrücklich hinstellt (l. c. S. 8. Einleitung) „die primäre, myopathische Natur der progressiven Muskelatrophie und deren rom Nervensystem unabhängige Genese darzulegen", an der Hand der durch unseren Sectionsbefund gegebenen Thatsachen zu prüfen, wozu die genaue Untersuchung sowohl des Rückenmarkes, namentlich aber auch der Wurzeln desselben eine nicht ungünstige Gelegenheit bietet.

Den Nachweis der secundären Natur der Veränderungen am Rückenmarke stützt Friedreich bekanntlich anf zwei Gründe; entweder die Veränderungen am Rückenmarke seien entzündliche und dann durch Fortpflanzung des entzündlichen Processes in den Muskeln hervorgerufen, oder sie seien einfach atrophischer Natur und die Folge der durch die Atrophie der Muskeln verursachten Inactivität derselben. Als entzündliche Veränderungen sieht nun Friedreich (l. c. S. 121) auch jene Zellenwucherungen um und im Centralcanal an, welche in einer Reihe von Fällen (Clarke und Rad cliffe, Hay e m, Duménil u. A.m.) 
angeführt werden, und bringt diese insofern mit dem entzündlichen Processe in den Muskeln in Verbindung, als er anführt (l. c. ibid.) "dass der entzündliche Process folgend dem die Faserzüge begleitenden, wenn auch nur sehr spärlichen Stroma, anf die Neuroglia der grauen Rückenmarkssubstanz sich forterstreckt, innerhalb derselben nach verschiedenen Stellen sich ausbreitet, um alsdann nicht selten an gewissen, von der Eintrittsstelle der vorderen Wurzeln entfernt gelegeneren, aber durch eine besondere Reichlichkeit der Neuroglia ausgezeichneten Stellen eine vorwiegende Höhe and Entwickelnng zu erreichen." In unserem Falle nun finden sich die von ihm angeführten Zellenanhäufungen an Stelle des Centralcanals oder um denselben in sehr bedentender Menge, allein sowohl die vorderen Wurzeln als auch das übrige Grau des Rückenmarkes zeigte sich vollkommen intact, so dass an ein Fortkriechen des entzündlichen Processes von den Muskeln aus nicht gedacht werden kann; aber, wie schon oben erwähnt, dürfte selbst die entzündliche Natur dieser Zellenanhäufungen nicht anzuerkennen sein, wenigstens nicht für die Mehrzahl der Fälle, zumal ja über das constante Vorkommen des Centralcanals, oder dessen Er. setztsein durch solche Zellenanhäufungen, die Histologen sich noch keineswegs geeinigt haben. (Siehe über die ganze Frage: Henle's Handbuch der Nervenlehre des Menschen. 1871. S. 43.) Meine eigenen Untersuchungen haben mich belehrt, dass diese Zellenanhäufungen an den verschiedensten Rückenmarken vorkommen, an denen sonst dnrchaus kein Zeichen von entzündlicher Reizung zu sehen ist. Allein auch die zweite Art der Erklärung, welche Friedreich anfübrt, dass die Veränderungen an den Ganglienzellen der Vorderhörner, dort, wo bloss solche gefunden werden, nur Folge der Inactivität der Muskeln sind, kann ich nicht als zutreffend anerkennen. Friedreich stützt sich nämlich auf die von Vulpian (Archives de physiol. norm. et path. 1868. p. 443 und 1869. p. 678 und p. 690), von Dickins on (Journal of Anatomy and Physiology. 1868. Nor.) und ron Clarke (Med. chir. Transactions Vol. 51. 1868) veröffentlichten Rückenmarksbefunde nach lange vorher stattgehabten Amputationen, die er für ganz identisch mit den bei der progressiven Muskelatrophie gefundenen erklärt; allein dies kann nicht zugestanden werden, da es sich in diesen Fällen um Atrophie entweder der ganzen betreffenden Rückenmarkshälfte oder des betreffenden Hornes handelte, nie aber bloss Veränderungen der grossen Ganglienzellen der Vorderhörner gefunden wurden, so dass es nicht zulässig erscheint, diese letztere Art des Befundes bei der progressiven Muskelatrophie mit jenen Veränderungen zu identificiren. 
Wenn sich Friedreich darauf beruft, dass Clarke in seinem Falle die Veränderungen an den Ganglienzellen ausdrücklich als identisch mit den bei der progressiven Muskelatrophie gefundenen bezeichnet, so zeigte doch derselbe Fall auch dentliche Reste entzündlicher Veränderungen der grauen Substanz; ausserdem steht Friedreich's Ansicht die positive Thatsache entgegen, dass nach apoplectischen Ergüssen im Gehirne nie Atrophie der Ganglienzellen der Vorderhörner eintritt, ausser in jenen seltenen Fällen des Uebergreifens der secundären (T ür ck'schen) Degeneration der Seitenstränge auf die Vorderhörner, wie sie Charcot (1. c. pag. 245) berichtet, in welchen sich dann eine rasch fortschreitende Atrophie der gelähmten Theile anschliesst.

Hatten wir bisher blos aus anatomischen Gesichtspunkten die Friedreich'sche Ansicht zu widerlegen versucht, so dürften sich doch auch klinische Thatsachen als dazu geeignet erweisen. Eine Reihe von Fällen, welche Friedreich (l. c. S. $217 \mathrm{ffg}$.) als rheumatische Formen der progressiven Muskelatrophie bezeichnet, sind durch nenere Forschungen als Fälle von Poliomyelitis anterior acnta ausgeschieden (Erb, Ueber acute Spinallähmung bei Erwachsenen. Dieses Archiv. V. Bd. S. 758), und auch Friedreich's Fall VI, (1. c. (S. 31) der sich durch fieberhaften Beginn, hochgradige, lä̉mungsartige Schwäche mit daran sich anschliessender partieller Atrophie auszeichnet, alles Symptome dieser klinisch gut characterisirten Form ron Kinderlähmung, deren Vorkommen bei Erwachsenen zuerst von D u chenne constatirt wurde, gehört hierher; über die spinale Natur des Leidens besteht um so weniger ein $Z$ weifel, als auch schon ein Sectionsbefund von Gombault vorliegt (Note sur un cas de paralysie de l'adulte suivi d'autopsie. Arch. de phys. 1873. p. 80). Allein auch die Aetiologie weist mehr auf das Rückenmark hin, und wenn Friedreich (1. c. S. 217) seiner Anschaung von der myopathischen Natur der progressiven Muskelatrophie zu Liebe zur Erklärung dieser rheumatischen Formen zu Hypothesen über die durch die Kälte herbeigeführten Ernährungsstörungen in den Muskeln greift, so ist der Einfluss der Kälte auf das Rückenmark experimentell durch $\mathbf{F}$ e in berg erwiesen (Le yd e $\mathrm{n}$, Klinik der Rückenmarkskrankheiten. 1874. I. Bd. S. 171), und auch die Aetiologie der Tabes dorsualis, welche in den weitaus meisten Fällen nicht, wie früher angenommen, in sexuellen Excessen, sondern in längerer hochgradiger Kälteeinwirkung zu suchen ist, kann als ein positiver Beweis dafür angeführt werden; es sind die Fälle nicht gar zu selten, in welchen sich unmittelbar an eine hochgradige Erkältung die ersten 
Erscheinungen der Tabos anschliessen, eine Erfahrung, welche namentlich Militärärzte zu machen öfter Gelegenheit habes.

Einen klinischen Einwand gegen die Zusammenstellung der progressiven Muskelatrophie mit der Kinderlähmung sieht Friedreich (l. c. S. 144) darin, dass „es unmöglich gestattet sein könne, Krankheiten, welche bezuiglich ihrer Entwickelungsweise, ihres Verlanfes und ihrer klinischen Symptomatologie so cardinale Differenzen darbieten, auf durchaus identische anatomische Veränderangen zurückzuführen"; allein es dürfte nicht schwer fallen, diesem Einwurfe durch Beispiele aus anderen Gebieten der Pathologie zn begegnen, um nachzuweisen, dass es sehr wohl denkbar, dass chronische und acute Processe zu denselben pathologischen Endproducten führen, was ja neuere Sectionsbefunde gerade für die von Friedreich einander gegenübergestellten Krankheiten, Kinderlähmung und progressive Muskelatrophie, nachweisen; allein selbst abgesehen davon, glaube ich, trotzdem die Vorgänge ron $\mathrm{Clarke}$ und $\mathrm{Charcot}$ (1. c. pag. 184. Note) theilweise schon schematisirt sind, dass wir bei dem gegenwärtigen Stande der Untersuchungsmethoden nicht im Stande sind, aus vorliegenden Bildern a posteriori Rückschlüsse auf die vorausgegangenen Processe an den Ganglienzellen zu machen, und keineswegs ausgeschlossen werden kann, dass differente Processe, acut entzündliche, chronisch irritative und einfach atrophische schliesslich zu denselben Veränderungen der Ganglien. zellen führen, eine Anschaunng, die ihren ganz präcisen Ausdruck in der Angabe Leyden's (1. c. S. 76) findet, der anf die bedeutende Schwierigkeit hinweist, die es macht, Atrophie und Sclerose der Ganglienzellen, also zwei sicherlich sehr differente Processe, genau zu scheiden; namentlich aber dürfte Friedreich's Verlangen (l. c. S. 137), als Beweis für den schleichenden Reizungsvorgang in den Ganglienzellen, für welchen Charcot u. A. die Veränderungen halten, Schwellung und Vergrösserung des Protoplasmas, Kerntheilung za finden, wohl zu weit gehen, indem solche Veränderungen bisher nur bei acuten, entzündlichen Processen vorgefunden wurden, so von $\mathrm{H}$ ayem (Note sur deux cas de myélite aiguè centrale et diffuse. Arch. de phys. norm. et path. 1874. Juli-Sept.) die Schwellung der Zellkörper, von Jolly (Ueber traumatische Encephalitis. 1869) die Kerntbeilung, welche letztere jedoch Leyden (1. c. II. Bd. 1. Abthlg. S. 130) nur sebr selten nnd nicht unzweifelhaft gesehen, während Charcot (l. c. pag. 184) dieselbe ganz leugnet.

Wenn noch Friedreich in mehreren Paragraphen seines Cap. V. Fälle von peripherischen Formen der Muskelatrophie beibringt, so 
können dieselben nichts anderes beweisen, als ihre eigene Existenz, die auch keineswegs geleugnet wird; als ein Beweis gegen den centralen Ursprung der progressiven Muskelatrophie können dieselben nicht gelten; auf die Frage des myopathischen oder neurotischen Ursprunges jener Formen kann hier nicht eingegangen werden.

Soll nun das Résumé der an den vorliegenden Fall geknüpften Erörterungen gezogen werden, so wäre dasselbe dahin zu präcisiren, das für denselben der Nachweis von der primären Natur der Veränderungen am Rückenmarke unzweifelhaft geführt ist.

Am Schlusse der Arbeit angelangt, erübrigt mir nur noch, Herrn Professor Westphal für die freundliche Ueberlassung sowohl der Krankengeschichte als des anatomischen Materiales meinen verbindlichsten Dank zu sagen.

Berlin, im October 1875. 